\title{
Performance analysis: improving the Dutch railway service
}

\author{
V. A. Weeda ${ }^{1,2}$ \& K. S. Hofstra ${ }^{2}$ \\ ${ }^{I}$ Delft University of Technology, Department of Transport \& Planning, \\ The Netherlands \\ ${ }^{2}$ ProRail Performance Analysis Office, Utrecht, The Netherlands
}

\begin{abstract}
Growth ambitions in railway transport make adequate capacity management essential. Although current planning rules are meant to support the construction of a feasible timetable, some rules fail to prevent conflicts and others even cause them. Detailed computer-aided performance analysis reveals these problems as occurring in real life. Feedback from operations to planning, in recent years explored and promoted by the Transport and Planning Department of Delft University of Technology, is now accommodated in the Performance Analysis Office of the Dutch railway infrastructure manager ProRail. Lessons learned from operations analyses give rise to advices in various stages of the planning process. Several improvements have been implemented in the past two years making the traffic process more smooth and predictable. Less variation requires smaller buffer times and allows more trains on the existing infrastructure. The paper will issue the opportunities and illustrate the current state of performance analysis by TU Delft and ProRail with recent cases.
\end{abstract}

Keywords: performance analysis, punctuality, capacity management.

\section{Introduction}

More trains and fewer delays are important targets for the government and the railway industry in the Netherlands. These make adequate capacity management increasingly important. In current practice, planning rules are supposed to guarantee feasibility. A look into real life with a more location-specific approach, however, enables a more efficient timetable. 
Adequate feedback from operations to planning requires accurate train records, which many common tools and databases lack. Detailed analysis of Dutch railway operations was pioneered and experimented on by Delft University of Technology, in which the data mining tool TNV-Prepare [1] has been essential. The level of detail was unprecedented, but convenience was an issue. The availability of a new data format enabled a more user-friendly way of data preparation. To this end, the railway industry developed the VTL-tool [4] that not only extracts train records but also yields new analysis possibilities. Using the same new format, TU Delft recently developed TNV-Conflict for an exact identification of route conflicts [2].

Two years ago, the Traffic Control department of Dutch railway infrastructure manager ProRail established the Performance Analysis Office in order to accommodate feedback from operations to planning in the organisation. Together with mainline services operator NS, the office identifies and addresses problems in railway operations. An important underlying principle is the awareness that only about $25 \%$ of dispuncuality can be attributed to incidents; the remainder is caused by a mismatch between timetable and daily operations. In the performance analyses, the VTL-tool plays a crucial role. Lessons learned give rise to advices in various stages of the planning process. A range of improvements has been implemented in real life already. This paper gives an impression of the current state of performance analysis with recent cases, regarding:

- evaluation of running time calculation;

- adequate allocation of margins;

- unravelling nodes;

- dynamic crossing;

- fine-tuning the automatic route setting.

\section{Evaluation of calculated running time}

Construction of a train path starts with calculated technical running times, taking infrastructure and rolling stock characteristics into account. The resulting figures serve as input for the scheduling tools. As a part of the control loop, the minimal running times are compared with records. On average, the 10-percentile of measured running times corresponds fairly well with the calculations. Certain types of rolling stock, however, appear to be faster in reality than in the planning tool. At least this holds for so-called IRM (double-stock inter-regio trains). This discrepancy was investigated at TU Delft on a line with a sufficient resolution of train running data, homogeneous rolling stock use and few conflicts.

Parallel to the coast, IC trains from The Hague (HS station) to Leiden are operated with IRM rolling stock and scheduled to cover the 15 kilometres in 11 minutes. The calculated running time is 10.1 minutes, while the operational 10 percentile is as short as 8.8 minutes. Speed profiles are reconstructed out of track section occupation data from May 2007 and section positions. Figure 1 shows 10-, 90- and in-between percentiles of train speeds, as well as the speed profile 
according to the minimal running time calculation. Small irregularities indicate the accuracy limits of the measurement method.

It can clearly be seen that real trains accelerate faster and keep a relatively high speed longer than calculations indicate, although coasting or braking starts somewhat earlier. A difference of approx. one minute between calculation and operations is regularly observed with IRM-run services between two stations. As long as the discrepancy is not resolved in the calculation tools, the recognition alone already brings opportunities for optimisation.

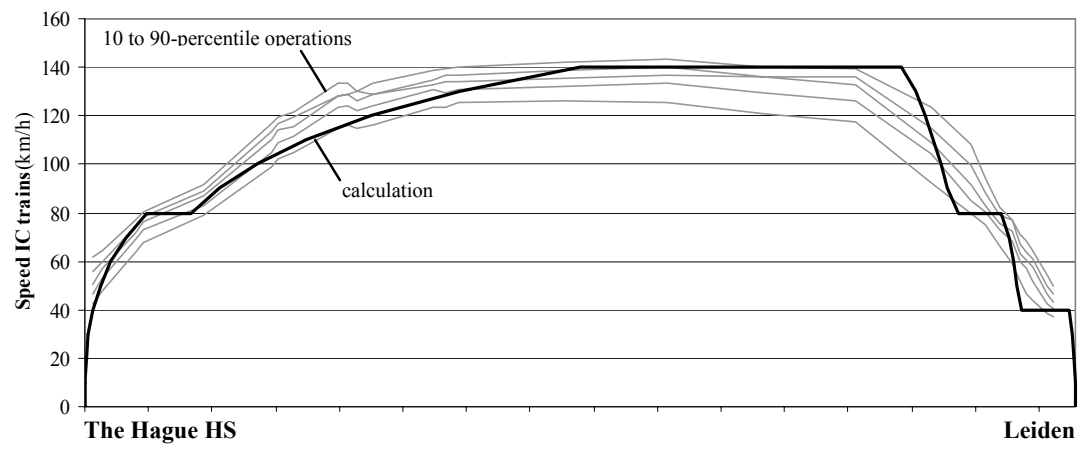

Figure 1: $\quad$ Calculated and measured speed profiles IRM rolling stock.

\section{Adequate allocation of margins}

A feasible timetable requires realistic running and dwell times. Current design standards prescribe a $5 \%$ supplement on top of calculated minimal running times, and a dwell time at line side stops of 0.5 up to 0.7 minute depending on rolling stock type. As earlier research by TU Delft revealed [6], these standards produce a feasible schedule for inter-city (IC) trains, but not for local trains. The latter is caused by a time-consuming departure procedure and variations in acceleration and braking behaviour around stops.

The problem shows up at various locations, usually drawn to the attention by a poor punctuality. A case recently worked on is found on the double-track line of Utrecht-Geldermalsen in the middle of the Netherlands. Current track usage has a heterogenic structure, with an IC train and a local train every quarter, and optional freight paths twice an hour. IC trains do not call at Geldermalsen. Although the timetable meets the standards, IC trains usually run into a conflict with local trains. One of the IC/local pairs is analysed here.

The local and IC train have scheduled running times from Utrecht to Geldermalsen of 20 and 15 minutes, respectively. These include line-side dwell times, and running time supplements according to the standard: the local train 1.8 minutes or $9.9 \%$ and the IC train 0.8 minutes or $5.6 \%$. In the planning, the IC train overtakes the local train 2 minutes after the latter has moved away from the main track to a platform track. This headway includes 1 minute of buffer time. 
During operations, a conflict occurs as visualised in Figure 2. Thick lines are the 2008 timetable and thin lines represent the empirical bandwidth of both trains, based on departure, through and arrival records from April 2008. The leftmost line of each grey bundle corresponds with early trains (10-percentile) and the rightmost line with late trains (90-percentile). It can be seen that:

- The local train hardly manages to operate within schedule and regularly arrives slightly late at Geldermalsen. This is in line with the observed excess of running and dwell times mentioned above.

- The IC train would autonomously pass through Geldermalsen ahead of schedule. That effect is even stronger because of a mismatch between theory and practice explained in Section 2. This particular train is run by IRM rolling stock and is faster in reality (13.6 minutes) than in the planning tools (14.2).

The IC train is often delayed by the late local trains: despite almost one minute of running time supplement, punctuality drops from $90 \%$ in Utrecht to $85 \%$ in Geldermalsen. It is important to note that the conflict is a consequence of the design standard prescribing a running time supplement for this IC train.

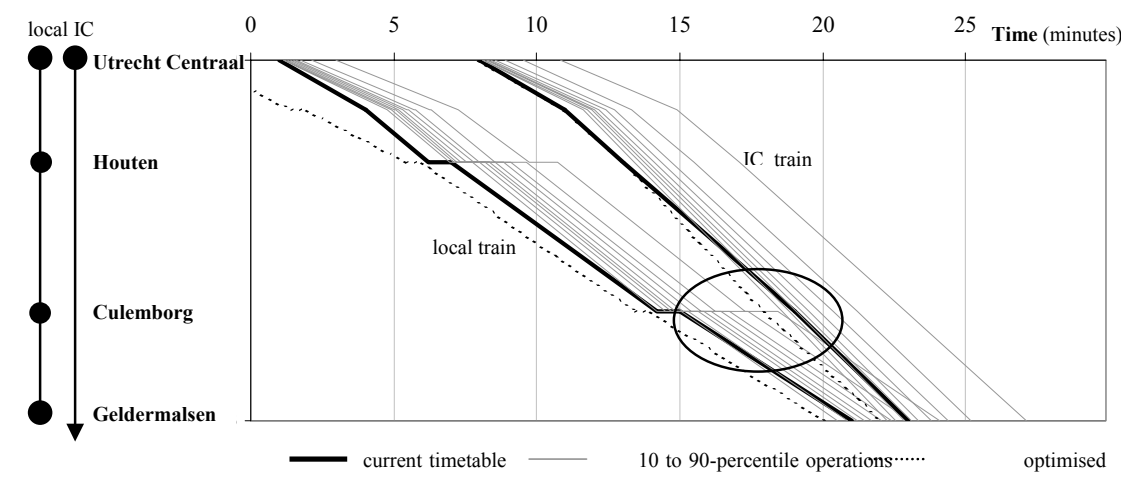

Figure 2: $\quad$ Schedule and empirical bandwidth of local and IC train UtrechtGeldermalsen.

Following these insights, an optimised timetable has been designed, drawn as two dotted lines in Figure 2. In the IC path, all running time supplement is shifted towards the end of the line, beyond Geldermalsen. This reflects the autonomous run of on time running trains: the fastest trains adhere to the path and trains do not pass early. Now, the 2 minutes overtaking headway requirement at Geldermalsen forces the local train to be advanced. This one is scheduled one minute earlier ( 3 minutes in Utrecht to fit in an additional stop). Effectively, the headway is enlarged by one minute, making the IC path more feasible. Since most trains only have a small delay, one minute of buffer time is very effective in reducing conflicts and thus improving punctuality. The optimised timetable will most probably be put into service by the end of 2008 . 


\section{Unravelling}

In main stations where various lines meet, the platform track occupation can be a challenging puzzle. Trains with different destinations may successively use the same points and platform tracks; headway times are usually the main solution criteria. This works as long as all trains run on time. Otherwise, however, the interdependencies between corridors make delays on one line affect trains on other lines.

A certain level of variations may be inevitable. It can be made acceptable though, if delays are isolated. The Performance Analysis Office therefore introduced the concept of Unravelling: tracks in main stations are assigned to independent corridors without crossing movements at grade. Busy lines require more than one platform track, but every platform track is used in only one corridor. This approach brings about two worthwhile benefits:

- When trains on one line arrive late, the other lines can still operate normally. Late trains pass the station smoothly in their own corridor without gathering additional delays.

- The decrease of crossing movements implies a lower track occupation with the same traffic flow, thus creating buffer time or capacity for additional traffic.

As usual in complicated scheduling tasks, there are new problems as well: sufficiently long (platform) headways may become an issue. In Utrecht, difficulties of that kind were limited to a tight headway for a freight path used a few times a day.

\section{Utrecht}

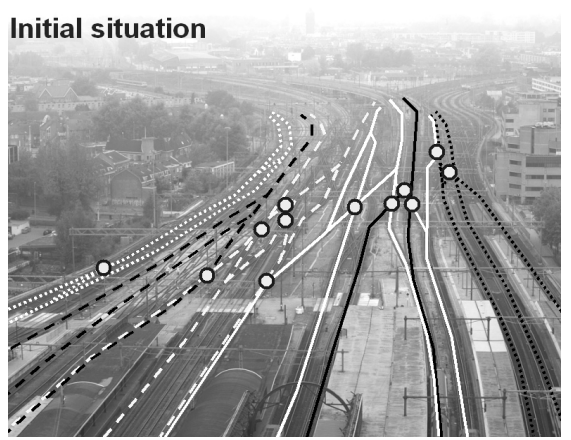

Utrecht

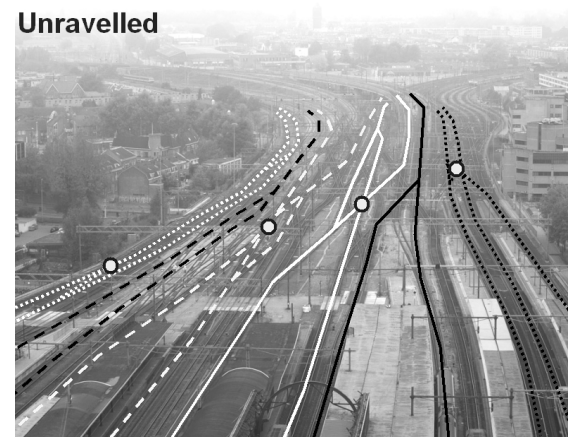

Figure 3: Track occupation Utrecht: reduction of crossing movements.

The concept of Unravelling has been successfully implemented in the Central Station of Utrecht. The original 2007 track occupation plan met headway and other requirements, but was vulnerable in case of delays because of dependencies between corridors on points and platform tracks. A symptom of this was that, e.g., $33 \%$ of the IC trains from Rotterdam faced a red signal before being able to enter the station.

An alternative plan was designed whilst preserving arrival and departure times and major cross-platform connections. The reduction of conflicting 
movements is visualised in Figure 3. Put into operation June 2007, the unravelled plan confirmed to be beneficial in several ways:

- Operational staff perceived smooth operations and considered a fixed track for each destination easy for passengers.

- Punctuality increased. The arrival punctuality at Utrecht was $2 \%$ higher in the second half of 2007 than in the first half year, while nationwide figures hardly changed.

- The number of stops before the station reduced by $14 \%$ over all trains, with a reduction up to $66 \%$ around the largest track plan changes. The latter applies to the corridor from the west (the light dotted line in the middle of Figure 3) which used to share platforms with other corridors. Now, only about $10 \%$ of the trains from Rotterdam have to make an unscheduled stop before arrival.

To this end, Unravelling seems to be an appropriate way to improve the quality of railway operations and enable further transport growth. Although the idea is quite straightforward and hence applied in many stations, it is not demanded by any design method or requirement. For a range of nodes, unravelled track occupation plans have been designed and implemented last two years, but at some stations there is still room for improvement.

\section{Dynamic crossing}

Various cases have illustrated that the current timetable design standards allow, or even cause conflicts in the operations. The optimisation efforts are mostly aimed at reducing these conflicts. Sometimes, however, performance can be improved by creating conflicts. An interesting example in recent ProRail practice is found in the south of the Netherlands.

In the Eindhoven area, two IC trains cross at grade (see Figure 4). The original plan let both trains await mutual passenger connections and scheduled departure times. They crossed after departure from Eindhoven in a fixed order: IC 800 first and 3 minutes later IC 1900. If either train arrived late, both left the station with delay. The departure headway was worsened by the departure procedure also mentioned in Section 3: after clearance of the signal it takes half a minute on average to close doors and start moving.

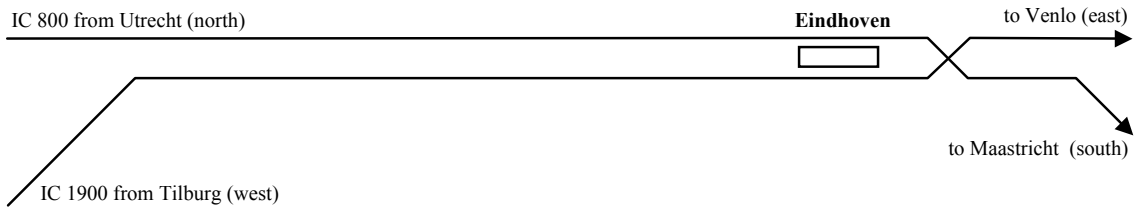

Figure 4: Crossing of IC lines in Eindhoven: original situation.

An adjusted regime has been constructed, where both trains cross earlier (at full speed points Liempde, see Figure 5) and dynamic, being scheduled almost at the same time. This is an element of dynamic traffic management [3]: the first 
coming train is first served. The other train might suffer a small delay but this will fade out during running and dwell time. Two dependencies (crossing conflict and passenger connection) have been separated and both trains leave Eindhoven independently.

The new concept was put into operation July 2007. The idea works: if one train arrives slightly late, both trains depart on time. Punctuality and percentage of successful passenger connections have substantially improved. Here, two trains run in the same direction, but the idea is also applicable to a junction with trains in opposite directions.

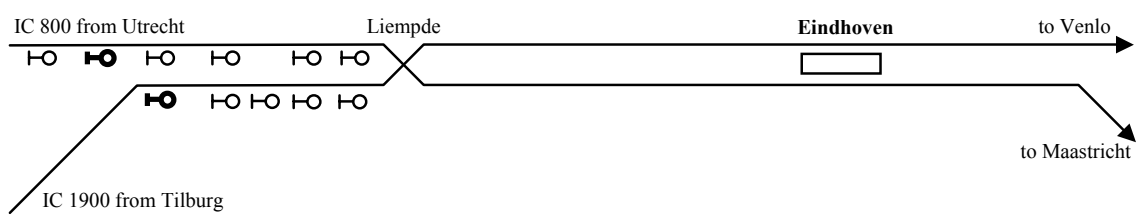

Figure 5: Crossing of IC lines in Eindhoven: dynamic situation.

\section{Fine-tuning the Automatic Route Setting}

Feedback does not end with a good planning. Performance analysis may also uncover opportunities to improve operational settings. Dutch railway traffic management works with Automatic Route Setting (ARS). Train dispatchers supervise this process and may intervene, but the majority of routes are automatically set. Therefore, the ARS settings play a key role in the delivered quality of operations. An important "control button" is the position of so-called triggers (an ARS trigger is the passing location that initiates points and signals further ahead to be set). Effects of suboptimal settings include unwanted order changes and unnecessarily restrictive signal aspects. These effects can be evaluated with operational data on the level of section occupation and release times. The use of detailed measurements of local headways for optimising the traffic process has been investigated and promoted by TU Delft in several publications $[5,7]$.

The opportunities for ARS fine-tuning can well be demonstrated on the case in the previous section (see Figure 5). Upon the implementation of dynamic crossing at Liempde, train orders and conflicts were analysed for a better insight with data from September 2007. The position of ARS triggers for the points at Liempde are drawn as bold signals in Figure 5. Both IC's are scheduled at the same time at these trigger locations. In Figure 6, the sharp separation line of the light and dark dots shows that "first come first served" works. Each train is served first in approx. $50 \%$ of the cases. In other words, both trains are given the same priority.

The time loss of the secondly served train is measured by determining running times from the ARS trigger up to a measuring point far enough to include re-acceleration. This shows that $70 \%$ of both trains suffers no delay or less than 1 minute. The average worst case delay (i.e. both trains pass the ARS 
trigger more or less at the same time) is 2.4 minutes for IC 800 and 2.1 minutes for IC 1900. This can be understood because IC 1900 has a speed restriction due to a curve, so it has to re-accelerate anyway. The minimal headway time between both trains is 1.8 minute at Liempde regardless of the order, and slightly increases further down the line due to re-acceleration. This is in line with the worst case delay of just more than 2 minutes.

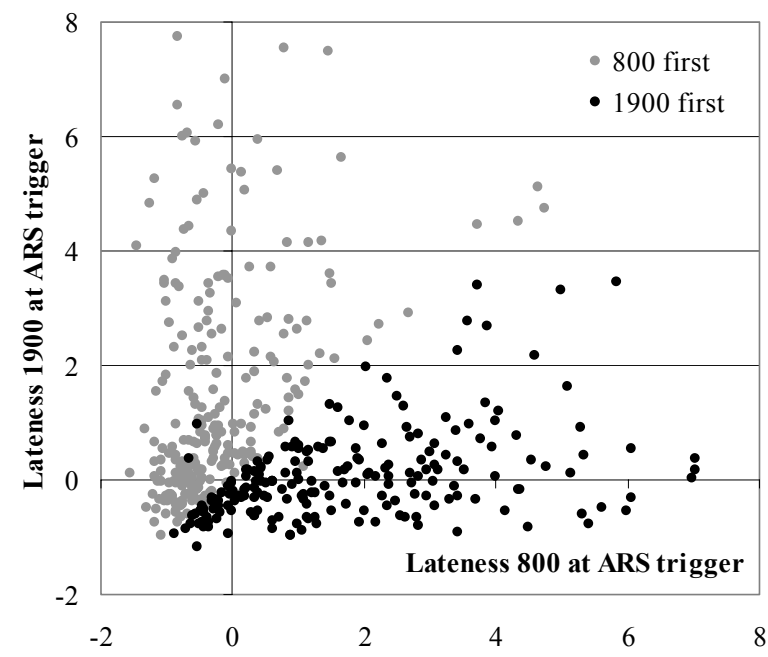

Figure 6: Order of trains at Liempde points.

Although the concept of dynamic crossing worked out well right away, the ARS could be fine-tuned. For several reasons it was considered better to make a priority shift in favour of IC 800 :

- The worst case delay is smaller for IC 1900 than for IC 800 .

- On its track, IC 1900 has a certain risk of meeting a local train further ahead, so being early does not pay off.

- IC 800 still has a lower punctuality in Eindhoven than IC 1900.

- Because of its fixed speed restriction, IC 1900 needs less additional energy to re-accelerate after a conflict.

The priority shift was carried out in October 2007 by moving the ARS trigger of IC 800 one position upstream (the leftmost signal in figure). This made the IC 800 claim it's route approx. 45 seconds earlier. A second analysis showed that under the new ARS settings, IC 800 gets the route first in $67 \%$ of the cases, compared to $50 \%$ initially. The quick implementation and visible effect of this kind of fine-tuning illustrate the current command over operations.

\section{Conclusions}

Performance analysis is developing from an academic field of study to an essential part of railway traffic and capacity management. Detailed evaluations 
by means of advanced computer software close the control loop from operations to planning. Improvements as the ones described have been implemented during 2007 and 2008 throughout the country and reportedly make the traffic process smooth and predictable. Often, seemingly small changes have considerable benefits. Less variation requires smaller buffer times and allows more trains on the existing infrastructure. The successful practice-based approach is recognised by a growing part of the railway industry.

So far the adjustments had the nature of local specific work. For further incorporation in the regular planning process, the Performance Analysis Office seeks to capture the philosophies detailed in this paper in improved design standards or rules of thumb. Nevertheless, local specific operational analysis will remain important, for the result should be leading.

\section{References}

[1] Goverde, R.M.P. \& I.A. Hansen, "TNV-Prepare: Analysis of Dutch railway operations based on train detection data". In: J. Allen et al, Computers in Railways VII, WIT Press, Southampton, 2000, pp. 779-788

[2] Goverde, R.M.P., W. Daamen \& I.A. Hansen, "Automatic identification of route conflict occurrences and their consequences". Ibid.

[3] Schaafsma, A.A.M., "Dynamic Traffic Management - innovative solution for the Schiphol bottleneck 2007”. In: Proceedings $1^{\text {st }}$ International Seminar on Railway Operations Research, Delft, 2005.

[4] Stam-Van den Berg, B.W.V. \& V.A. Weeda, "VTL-Tool: Detailed Analysis of Dutch Railway Traffic". In: Proceedings $2^{\text {nd }}$ International Seminar on Railway Operations Research, Hannover, 2007.

[5] Tromp, J.P.M. \& I.A. Hansen, "Hindrance between conflicting train movements at a level crossing". In: J. Allen et al, Computers in Railways VIII, WIT Press, Southampton, 2002, pp. 985-993

[6] Weeda, V.A. \& P.B.L. Wiggenraad, "Joint design standard for running times, dwell times and headway times". In: J. Allen et al, Computers in Railways X, WIT Press, Southampton, 2006, pp. 611-620

[7] Yuan, J. \& I.A. Hansen, "Analysis of scheduled and real capacity utilisation at a major Dutch Railway station". In: J. Allen et al, Computers in Railways IX, WIT Press, Southampton, 2004, pp. 593-602 